\title{
LINKAGE ANALYSIS OF FIRST SIX MODY GENES TO KNOWN LOCI AND SCREENING OF THREE MOST PREVALENT MODY GENES IN PAKISTANI POPULATION
}

\author{
ATTIYA K.$^{1^{*}}$, SAHAR F. ${ }^{2}$, ISMAIL M. ${ }^{3}$, QAISAR M. ${ }^{4}$ and AMARA J.* \\ ${ }^{1}$ Department of Computer Sciences and Bioinformatics, Mohammad Ali Jinnah University, Sihala \\ 2Institute of Biomedical and Genetic Engineering (IBGE), Islamabad \\ * Corresponding author: Email- attiya_kanwal@yahoo.com
}

Received: June 15, 2011; Accepted: June 24, 2011

\begin{abstract}
The pandemic of metabolic disorders is accelerating in the urbanized world posing huge burden to health and economy. The key pioneer to most of the metabolic disorders is insulin dependent diabetes mellitus or Type 1 Diabetes Mellitus (T1DM) and non-insulin dependent diabetes mellitus commonly known as Type 2 Diabetes Mellitus (T2DM). Both of these forms of diabetes are polygenic and multifactorial. A newly discovered form of diabetes is Maturity Onset Diabetes of the young (MODY). MODY is a monogenic form of diabetes and it is inherited as autosomal dominant disorder. Its age of onsets is at 14-25 years in individuals who have a strong family history of Type 2 Diabetes Mellitus (T2DM). In most of the MODY patients genes involved in mediating and controlling the expression of insulin are concerned. Till date eleven genes that cause MODY have been identified all over the world. But in Pakistan no genetic research has been done on MODY. The aims of the present study were to examine the prevalence and nature of mutation in three common MODY genes on the basis of their occurrence in European populations. These three MODY genes are HNF4-Alpha, GCK, and HNF1-Alpha. 22 members spanning four generations and 11 affected with MODY in Pakistani population were examined. To determine the linkage or exclusion study of the family to six known loci for MODY, a minimum of two micro satellite markers each of the candidate regions of these loci for MODY were genotyped in all the available individuals of this family. To identify genes involved in susceptibility to MODY and to discover new genes and mutations contributing to MODY in Pakistani population, Single Strand Conformation Polymorphism technique (SSCP) was used. Our findings highlighted that those unidentified MODY genes that facilitate to cause this form of diabetes in European population may play a central role for diabetes characterized by autosomal dominant transmission in Pakistani population.
\end{abstract}

Key words: MODY- Maturity onset diabetes of the young, T1DM- Type 1 Diabetes Mellitus, T2DM- Type 2 Diabetes Mellitus, and SSCP- Single strand conformational polymorphism.

\section{INTRODUCTION}

MODY is the term that relies on the old classification of diabetes into juvenile-onset and maturity-onset diabetes. Tattersall (U.K.) and Fajan (U.S.A.) first described MODY in 1974 [1], after taking into account a group of young people with diabetes who were treated without insulin 2 years or more after diagnosis. Since the 1970s there has been great interest in MODY as it is a genetic form of diabetes. MODY is an ancestral form of early-onset type2 diabetes. It is a monogenic form of diabetes mellitus inherited in autosomal dominant mode $[2,3])$. It is primarily an outcome of impaired Beta-Cells of pancreas [2, 3]. MODY is not a single entity but represents genetic, metabolic, and clinical heterogeneity [4]. It generally develops in middle age, and mainly coupled with primarily scantiness of insulin secretion [5]. Till 2009 eight discrete MODY genes have been acknowledged [6]. These are the genes including HNF4A, encoding hepatocyte nuclear factor 4 Alpha [7], GCK, encoding glucokinase [8], HNF1A, encoding hepatocyte nuclear factor 1 Alpha [9], IPF1, encoding insulin promoter factor 1 [10], HN F1B, encoding hepatocyte nuclear factor 1 Beta [11], NEUROD1, encoding neurogenic differentiation 1 [12], KLF11, encoding for kruppel-like factor 11 [13] and CEL, encoding carboxyl-ester lipase [14]. Now four further genes have been exposed that cause MODY. These genes are PAX4, encoding, Paired Domain gene 4 (OMIM 612225), IPF1, encoding, Insulin Promoter Factor 1 (OMIM 606392), INS, encoding, Insulin (OMIM 176730) and BLK, encoding, Tyrosine kinase, B-Lymphocyte (OMIM 191305).

The true relative prevalence of the eleven distinct MODY subtypes is unknown and varies substantially in different populations [15, 16, and 17]. Mutations in the genes encoding HNF1A and GCK are by far the most prevalent. Mutations in GCK (MODY 2) account for $7-41 \%[15,16]$, whereas mutations in TCF1 (MODY 3) may account for 11-63\% [17] of mutations in subjects with clinically diagnosed MODY. Mutations in HNF4A (MODY1) are less frequent and may 
account $2-5 \%$ of subjects with MODY [17]. The prevalence of MODY patients with mutations in TCF2 (MODY 5) is considered very rare. It may comprise up to approximately $2 \%$ [18]. Mutations in IPF1 (MODY 4), NEUROD1 (MODY 6), KLF11 (MODY 7), CEL (MODY 8) and PAX4 (MODY 9) are also very rare and have been identified only 1\% [19]. The prevalence of MODY patients with mutations in INS (MODY 10) and BLK (MODY 11) are also considered only 1\% [19]. The aims of the present study were to elucidate the relative prevalence of the MODY subtypes caused by mutations in the genes HNF4A, GCK, and HNF1A, respectively, in a Pakistani population with strict clinically defined MODY.

\section{METHODOLOGY}

Ascertainment of families

Family affected by Maturity-onset diabetes was obtained through personal contacts. As an initial step, the family history was obtained from the representative members of the family and pedigree was draw. The extensive clinical examination of both patients and their unaffected normal relatives will carry out by expert clinician.

Pedigree drawing was performed using the Cyrillic (v2.10) program for genetic implication by the standard method described by [20]. The exact genealogic relationships for all the affected individuals were obtained through the extensive personal interviews of elders of the families. Figure 1 shows the pedigree of the family that is clinically diagnosed with MODY.

\section{Collection of blood samples}

Blood samples were drawn with informed consent from all members of the family by 5 c.c. syringes in $10 \mathrm{ml}$ (to be checked) Vaccutainer tubes (Becton Dickinson, Franklin Lakes, and NJ.) containing acid citrate dextrose (ACD) or heparin. The blood was stored at $4^{\circ} \mathrm{C}$ until DNA extraction.

\section{Extraction of Genomic DNA}

Genomic DNA was extracted from blood samples using standard methods known as Phenol chloroform method or organic method. This method of DNA extraction from peripheral blood lymphocytes involves 3 days procedure.

\section{Primer Designing}

A primer is a short synthetic oligonucleotide which is used in many molecular techniques from PCR to DNA sequencing. Specific primers for region of interest in genomic DNA were designed at the Institute of Biomedical and Genetic engineering (IBGE), Islamabad using Bioinformatics Primer Designing Tool Primer3 (http://primer3.sourceforge.net/). These primers were then synthesized commercially (Operon, Germany).
Following three genes were genetically screened for mutation detection:
1. HNF4a (MODY 1)
2. GCK (MODY 2)
3. HNF1a (MODY 3)

The table 1 shows the primers for the coding regions of these three genes.

\section{Genotyping}

To determine the linkage or exclusion study of the family to six known loci for MODY, a minimum of two microsatellite markers each of the candidate regions of these loci for MODY were genotyped in all the available individuals of this family. Microsatellite Markers used for genotyping this family were selected from Marshfield Map of STRPs set Version 8 available at IBGE.

Table 2 shows the Microsatellite Markers that are used to test linkage to known loci of MODY.

\section{Polymerase chain Reaction}

Standard PCR reactions will be performed using Bioline or Promega products as illustrated by Batzer et al., (1996) to amplify the DNA. PCR reactions were carried out on $\sim 40 \mathrm{ng} / \mathrm{ul}$ of DNA in different reaction volumes. A typical reaction mixture consisted of $1 \mathrm{ul}$ 10X PCR buffer [100mM Trizma-HCL, pH 8.3; 500mM $\mathrm{KCL} ; 15 \mathrm{mM} \mathrm{MgCl} 2 ; 0.01 \%$ (w/v) gelatin )(Bioline)], 2mM dNTPs (Promega), 20uM Forward and reverse primers (Operon, Germany), 5U/ul ul of Taq DNA polymerase (Institute of Biomedical and genetic engineering, Islamabad, Pakistan, Fermentas) and autoclaved deionized water. Each PCR reaction will be consistently performed using the GeneAmp PCR system 9700 thermocycler (Applied Biosystems, foster city, California, USA).

The amplifications conditions of each marker along with initial and final concentrations for required number of reactions are shown in the table 3.

\section{Polyacrylamide Gel electrophoresis}

Denaturing polyacrylamide gel electrophoresis technique will be employed to distinguish the different alleles of the polymorphic microsatellite markers used in linkage/homozygosity analysis. The standard apparatus used (Bio-Rad Sequi-Gen Cell) measured 38 * $50 \mathrm{~cm}$ and utilized thick spacers. . Amplified products were detected by placing the gel on UV transilluminator (Syngene Using GeneScan, ver 3.1.2 software, Cambridge, UK), (UV light of wavelength $254 \mathrm{~nm}$ ) and gel photographs were analysed to determine alleles.

\section{Mutation Detection}

To identify genes involved in susceptibility to MODY and to discover new genes and mutations contributing to MODY, following three genes for four different types of MODY will be screened for mutation detection:

\section{HNF4a (MODY 1)}




\section{GCK (MODY 2)}

3. HNF1a (MODY 3)

Mutation detection in any of the genes will be done through Single Strand Conformation Polymorphism technique.

Prior to SSCP we must set the condition and annealing temperature of each exon. Optimization of exons was done by polymerase chain reaction. Amplification was done in a final volume of $15 \mu \mathrm{l}$ containing 40ng genomic DNA, 1X PCR buffer (Bioline), $0.45 \mathrm{mM} \mathrm{MgCl} 2,200 \mu \mathrm{M}$ dNTPs (Promega) $1 \mu \mathrm{M}$ each forward and reverse primer, $1 \mathrm{U}$ Taq DNA polymerase. Amplification was done with initial denaturizing at $94^{\circ} \mathrm{C}$ for $2 \mathrm{~min}, 35$ cycles each consisting of denaturation at $94{ }^{\circ} \mathrm{C}$ for $45 \mathrm{~s}$, annealing for $45 \mathrm{~s}$, extension at $72^{\circ} \mathrm{C}$ for $45 \mathrm{~s}$ and final extension for 10 min at $72^{\circ} \mathrm{C}$. Forward and reverse primers for each exon with specific sequence were used in amplification. Amplified products were run on $2 \%$ $(\mathrm{w} / \mathrm{v})$ agarose gel containing $0.5 \mu \mathrm{g} / \mathrm{ml}$ ethidium bromide at constant power supply of 200 volts for 30 minutes.

\section{AGROSE GEL CASTING}

The 2 percent agarose gel was prepared by adding 6 gms of agarose in $300 \mathrm{ml} 1 \mathrm{X}$ TBE buffer (Tris Borate EDTA). Five $\mu$ l of Orange $G$ dye was added to $5 \mu \mathrm{l}$ Amplified DNA sample (PCR product) and this mixture was loaded in wells using a micropipette. The $100 \mathrm{bp}$ ladder (Fermentas) was also loaded in a well for size reference.

After the gel was loaded, it was placed in a gel tank i.e. Maxicell electrophretic gel system filled with $1 \mathrm{X}$ TBE buffer and the negative and positive indicator on the cover and apparatus chamber were properly oriented. In order to visualize the DNA, the gel was placed on a transilluminator (Syngene, Cambridge, UK), (UV light of wavelength $254 \mathrm{~nm}$ ).

\section{Single Strand conformation polymorphism}

Single-strand conformation polymorphism (SSCP), or single-strand chain polymorphism, is defined as conformational difference of single-stranded nucleotide sequences of identical length as induced by differences in the sequences under certain experimental conditions. This property allows distinguishing the sequences by means of gel electrophoresis, which separates the different conformations. A single nucleotide change in a particular sequence, as seen in a double-stranded DNA, cannot be distinguished by electrophoresis, because the physical properties of the double strands are almost identical for both alleles. After denaturation by formamide, single-stranded DNA undergoes a 3dimensional folding and may assume a unique conformational state based on its DNA sequence. The difference in shape between two single-stranded DNA strands with different sequences can cause them to migrate differently on an electrophoresis gel, even though the number of nucleotides is the same, which is, however, a shortfall of SSCP.

\section{RESULTS}

The unidentified MODY genes that facilitate to cause Maturity-onset diabetes of the young in European population may play a central role for diabetes characterized by autosomal dominant transmission in Pakistani population. From this research study it is concluded that the examined Pakistani family which is clinically diagnosed with MODY may be due to mutation in other MODY genes except the genes that are screened in the present research study for this family. The results indicate that there is no linkage to the known loci of MODY which are used for linkage in this study. Similarly through genetic screening the following findings come to our acknowledge that no mutation is indicated in this examined family in MODY genes. There may be some environmental factors that are involved in causing this disease in this family or may be this disease is due to mutation in other reported MODY genes that are not screened in this study.

\section{DISCUSSION}

In the present research work we aim to first find out the linkage of the most common MODY genes, HNF4a (MODY 1), GCK (MODY 2), HNF1a (MODY 3) to the known loci of MODY by using Microsatellite Markers. Our results of Exclusion study shows that there is no linkage of these known loci to this family that is clinically diagnosed with MODY. Our second objective after doing exclusion study or linkage analysis was to discover new genes or mutation other than known eleven genes or mutations that are reported so far. For this purpose we use the Single Strand Conformation polymorphism. Our findings highlighted that those unidentified MODY genes that facilitate to cause this form of diabetes in European population may play a central role for diabetes characterized by autosomal dominant transmission in Pakistani population.

\section{ACKNOWLEDGEMENTS}

I am feeling proud to articulate some obsession about my honorable research supervisors Dr. Sahar Fazal, Assitant Professor, Mohammad Ali Jinnah University, Sihala Campus and Mr. M. Ismail, Deputy Director and Mr.Qaisar Masood, Scientific Officer, Institute of Biomedical and Genetic Engineering, Islamabad. Countless thanks to for their indispensable moral support and scholastic enthusiasm during my whole study.

\section{DISCLOSURE}

All the authors contributed equally in this research study. 


\section{References}

[1] Tattersall R.B., Fajans S.S. (1975) Diabetes. 24:44-53.

[2] Anna L.Gloyn, Sian Ellard, Maggie Shepherd, Rodney T. Howell, Elizabeth M.Parry, Andrew Jefferson, Elaine R. Levy and Andrew T. Hattersley (2002) DIABETES. 51: 2329-2333

[3] Owen K., Httersley A. (2001) Best Practice and Research Clinical Endocrinology and Metabolism. 15: 309-323.

[4] Costa A., Bescos M., Velho G., Chevre J., Vidal J., Sesmilo G., Bellanne-Chantelot C., Froguel P., Casamitjana R., Rivera-Fillat F., Gomis R. and Conget I. (2000) European Journal of Endocrinology. 142: 380-386.

[5] Vaxillaire M., Froguel P. (2006) Endocrinol Metab Clin North Am 35: 371-384.

[6] Maciej Borowiec, Chong W. Liew, Ryan Thompson, Watip Boonyasrisawat, Jiang $\mathrm{Hu}$, Wojciech M. Mlynarski, Ilham El Khattabi, Sung-Hoon Kim, Lorella Marselli, Stephen S. Rich, Andrzej S. Krolewski, Susan Bonner-Weir, Arun Sharma, Michele Sale, Josyf C. Mychaleckyj, Rohit N. Kulkarni and Alessandro Doria (2009) PNAS. 106:

[7] Yamagata K., Furuta H., Oda N., Kaisaki P.J., Menzel S., Cox N.J., et al. (1996) Nature. 384: 458-60.

[8] Froguel P., Zouali H., Vionnet N., Velho G., Vaxillaire M., Sun F., Lesage S., Stoffel M., Takeda J., Passa P., et al. (1993) N Engl J Med 328: 697-702.

[9] Yamagata K., Oda N., Kaisaki P.J., Menzel S., Furuta H., Vaxillaire M., Southam L., Cox R.D., Lathrop G.M., Boriraj V.V., Chen X., Cox N.J., Oda Y., Yano H,. Le Beau M.M., Yamada S., Nishigori H., Takeda J., Fajans S.S., Hattersley A.T., Iwasaki N., Hansen T.,
Pedersen O., Polonsky K.S., Bell G.I., et al. (1996) Nature 384:455-458.

[10] Stoffers D.A., Ferrer J., Clarke W.L., Habener J.F. (1997) Nat Genet. 17:138139.

[11] Horikawa Y., et al. (1997) Nat Genet 17:384-385.

[12] Malecki M.T., Jhala U.S., Antonellis A., Fields L., Doria A., Orban T., Saad M., Warram J.H., Montminy M., Krolewski A.S. (1999) Nat Genet. 23:323-328.

[13] Neve B., Fernandez-Zapico M.E., Ashkenazi-Katalan V., Dina C., Hamid Y.H., Joly E. et al. (2005) Proc Natl Acad Sci U S A. 102: 4807-12.

[14] Helge Ræder, Stefan Johansson, Pål I Holm, Ingfrid S Haldorsen, Eric Mas, Véronique Sbarra, Ingrid Nermoen, Stig § Eide, Louise Grevle, Lise Bjørkhaug, Jørn V Sagen, Lage Aksnes, Oddmund Søvik, Dominique Lombardo, Anders Molven \& Pål Rasmus Njølstad (2006) Nat Genet. 38: 5462.

[15] Gragnoli C. (2001) Diabetologia 44:13261329.

[16] Barrio R., Bellanne-Chantelot C., Moreno J.C., Morel V., Calle H., Alonso M., Mustieles C. (2002) J Clin Endocrinol Metabolism. 87: 2532-2539.

[17] Pruhova S., Ek J., Lebl J., Sumnik Z., Saudek F., Andel M., Pedersen O., Hansen T. (2003) Diabetologia. 46:291-295.

[18] Martine Vaxillaire and Philippe Froguel (2008) Endocrine Reviews. 29:254-264

[19] McCarthy M.I., Hattersley A.T. (2008) DIABETES. 57: 2889-98.

[20] Bennett R.L., Steinhaus K.A., Uhrich S.B., O'Sullivan C.K., Resta R.G., Lochner-Doyle D., Markel D.S., Vincent V., Hamanishi J. (1995) Am J Hum Genet. 56: 745-752. 
Table 1- Specific primers 5' to 3' sequences of above mentioned four genes for region of interest in genomic DNA

\begin{tabular}{|c|c|c|c|}
\hline \multicolumn{4}{|c|}{ HNF1ALPHA } \\
\hline Primer Name & Primer Sequence & Primer Size & Product size \\
\hline HNF1A_Ex 1F & TGCAAGGAGTTTGGTTTGTG & 20 & \multirow[t]{2}{*}{857} \\
\hline HNF1A_Ex 1R & TGGGGACTCAACTCAGAAGG & 20 & \\
\hline HNF1A_Ex 2F & CCTCAGGGTTGACAAGGTTC & 20 & \multirow[t]{2}{*}{771} \\
\hline HNF1A_Ex 2R & CAGCCCCCACCTATGAGTTA & 20 & \\
\hline HNF1A_Ex 3F & AAGAATCAAGGGCAAGGTCA & 20 & \multirow[t]{2}{*}{771} \\
\hline HNF1A_Ex 3R & GGCAACTGGACAGCCTTTTA & 20 & \\
\hline HNF1A_Ex 4F & CTCAGAACCСТCСССТTCAT & 20 & \multirow[t]{2}{*}{771} \\
\hline HNF1A_Ex 4R & GGACAGTCCTCCCCAACC & 18 & \\
\hline HNF1A_Ex 5F & TTTGAAGTGCTGAGGGCTGT & 20 & \multirow[t]{2}{*}{599} \\
\hline HNF1A_Ex 5R & CCAGAATCTCCCTGCCAAG & 19 & \\
\hline HNF1A_Ex 6F & CCTAGGGAGGCCCTGTGG & 18 & \multirow[t]{2}{*}{857} \\
\hline HNF1A_Ex 6R & CCACAGAATTGATGTATTATGCTT & 24 & \\
\hline HNF1A_Ex 7F & CTCTGGGAAGGAGAGGTGGT & 20 & \multirow[t]{2}{*}{771} \\
\hline HNF1A_Ex 7R & CGTGCACACACACAGATACG & 20 & \\
\hline HNF1A_Ex 8F & AGTCTTGAGGCCTGGGACTA & 20 & \multirow[t]{2}{*}{685} \\
\hline HNF1A_Ex 8R & CAGAGCAGCCTCCTGAGC & 18 & \\
\hline HNF1A_Ex 9F & GTGACAGAGCCCCTCACC & 18 & \multirow[t]{2}{*}{684} \\
\hline HNF1A_Ex 9R & ACAGTGACGGACAGCAACAG & 20 & \\
\hline HNF1A_Ex 10.1F & TGAGTACCCCTAGGGACAGG & 20 & \multirow[t]{2}{*}{855} \\
\hline HNFE1A_Ex 10.1R & AAGACTGTATCCCACGAAGCA & 21 & \\
\hline HNF1A_Ex 10.2F & ATCAGAAAGGGAGGGCTCTG & 20 & \multirow[t]{2}{*}{858} \\
\hline HNF1A_Ex 10.2R & CATGATGCTCTTGGGAACAA & 20 & \\
\hline HNF1A_Ex 10.3F & TTCCTGGGTGGCTACTCTGT & 20 & \multirow[t]{2}{*}{1030} \\
\hline HNF1A_Ex 10.3R & GGTTCACAGGCTCCTTTGTC & 20 & \\
\hline \multicolumn{4}{|c|}{ HNF4ALPHA } \\
\hline Primer Name & Primer Sequence & Primer Size & Product size \\
\hline HNF4A_Ex 1F & ACTCACCGCCTTCCTGGT & 18 & \multirow[t]{2}{*}{770} \\
\hline HNF4A_Ex 1R & TAAGAAACACACGGGGAGGT & 20 & \\
\hline HNF4A_Ex 2F & CСТССАССССТTCACAGAG & 19 & \multirow[t]{2}{*}{771} \\
\hline HNF4A_Ex 2R & CCAAAGATCTGCTCCTGGAC & 20 & \\
\hline HNF4A_Ex 3F & CTCTGTTGTTGCCACTCACC & 20 & \multirow[t]{2}{*}{771} \\
\hline HNF4A_Ex 3R & CTGCССССТСТССТСТGА & 18 & \\
\hline HNF4A_Ex 4F & AGGTGATGGAGTGGGAACAG & 20 & \multirow[t]{2}{*}{857} \\
\hline HNF4A_Ex 4R & TCTGGGACCTACCCACTCAG & 20 & \\
\hline HNF4A_Ex 5F & GGGCCCTAGTTCTGTCCTAA & 20 & \multirow[t]{2}{*}{599} \\
\hline HNF4A_Ex 5R & GTGTGGCTACAGTTGTAAATGACT & 24 & \\
\hline HNF4A_Ex 6F & AGAATGGAGGTGGAGGAGGT & 20 & \multirow[t]{2}{*}{771} \\
\hline HNF4A_Ex 6R & CССАСТССТCАTCAGTCACA & 20 & \\
\hline HNF4A_Ex 7F & CTGTGCAGGGGACAGAGAGT & 20 & \multirow[t]{2}{*}{857} \\
\hline HNF4A_Ex 7R & CCACGGCTATATCCCAGGT & 19 & \\
\hline HNF4A_Ex 8F & CAGCGTCACTGAGTTGGCTA & 20 & 599 \\
\hline HNF4A_Ex 8R & GCCACCATGTGAATCTCCTT & 20 & \\
\hline HNF4A_Ex 9F & CCACAGGCACCAGCTATCTT & 20 & 685 \\
\hline HNF4A_Ex 9R & AAATGAAAACGGCCTCTCCT & 20 & \\
\hline HNF4A_Ex 10F & TCTTGACTCCCCAGATGCTC & 20 & 855 \\
\hline HNF4A_Ex 10R & TCGGTGCCTGAACAAATAGA & 20 & \\
\hline HNF4A_Ex 11F & AGGAAGATGGCGTCCCAAG & 19 & 683 \\
\hline HNF4A_Ex 11R & САСТTTCTCCTGССТССТGT & 20 & \\
\hline HNF4A_Ex 12.1F & CGGGCCTCTTCATTTACTCC & 20 & 940 \\
\hline HNF4A_Ex 12.1R & AAGGCCTCAAGTCATCTCCA & 20 & \\
\hline HNF4A_Ex 12.2F & GGGAGACCTCTACTGCCTTG & 20 & 858 \\
\hline
\end{tabular}




\begin{tabular}{|c|c|c|c|}
\hline HNF4A_Ex 12.2R & TCCCTGAAGAAGGAGGATGA & 20 & \\
\hline HNF4A_Ex 12.3F & TTCTCСАAСАССАССТСТСC & 20 & 858 \\
\hline HNF4A_EX 12.3R & AGGACCTCAGCCTGGTGTT & 19 & \\
\hline \multicolumn{4}{|c|}{$\begin{array}{l}\text { GCK } \\
\end{array}$} \\
\hline Primer Name & Primer Sequence & Primer Size & Product size \\
\hline GCK Ex 1.1F & GGTCACCATGACAACCACAG & 20 & \multirow[t]{2}{*}{771} \\
\hline GCK Ex_1.1R & GGTTCAGTCACAGTTTCCTCCT & 22 & \\
\hline GCK Ex_1.2F & GGAGAACATCCAGGCTCAGT & 20 & \multirow[t]{2}{*}{772} \\
\hline GCK Ex_1.2R & TCTGAGGCTCAAACAAACCA & 20 & \\
\hline GCK Ex_2F & TGTTACCCACATGGCCTACC & 20 & \multirow[t]{2}{*}{771} \\
\hline GCK Ex_2R & TGGTAATCTGCAAAACCAAGG & 21 & \\
\hline GCK Ex $3 F$ & ACCCTGGTGACCATGTCC & 18 & \multirow[t]{2}{*}{685} \\
\hline GCK Ex_3R & GGCTTGGTGAAAGCGATAAG & 20 & \\
\hline GCK Ex_4F & GTGTGCAGATGCCTGGTG & 18 & \multirow[t]{2}{*}{685} \\
\hline GCK Ex 4R & CTGGCTGTGAGTCTGGGAGT & 20 & \\
\hline GCK Ex $5 \mathrm{~F}$ & ССТTCССТССТССТСТTTGT & 20 & \multirow[t]{2}{*}{685} \\
\hline GCK Ex_5R & CCACCCCTGGTAGACAGGT & 19 & \\
\hline GCK Ex_6F & GTCCCTGAGGAATAGCTTGG & 20 & \multirow[t]{2}{*}{685} \\
\hline GCK Ex_6R & GGGCTACATTTGAAGGCAGA & 20 & \\
\hline GCK Ex_7F & CCCTGTGCAGGAGGTAGTGA & 20 & \multirow[t]{2}{*}{597} \\
\hline GCK Ex_7R & AGGAGAAAGGCAGGCAGTG & 19 & \\
\hline GCK Ex_8F & GACGCTATCAAACGGAGAGG & 20 & \multirow[t]{2}{*}{684} \\
\hline GCKEx_8R & GCCCTTGAAGCCTGTTGTA & 19 & \\
\hline GCK Ex_9F & CCATTGTTCCAGACAAAGCA & 20 & \multirow[t]{2}{*}{771} \\
\hline GCK Ex_9R & CAAGCCCATTATCTGCAATG & 20 & \\
\hline GCK Ex_10F & GAGGGAAAGACGTGAACCAG & 20 & \multirow[t]{2}{*}{769} \\
\hline GCK Ex_10R & GCCCTGAGACCAAGTCTGC & 19 & \\
\hline GCK Ex_11F & CTCCCTGGAGAACGAGAGG & 19 & \multirow[t]{2}{*}{766} \\
\hline GCK Ex_11R & CTTGGGAACCGCAAGGAAC & 19 & \\
\hline GCK Ex_12.1F & CCGGTAATGAATGTGGAGGA & 20 & \multirow[t]{2}{*}{855} \\
\hline GCK Ex_12.1R & GGAAATTGATTCCAGCGAGA & 20 & \\
\hline GCK Ex_12.2F & ATACCGCTGGGGAACAGAG & 19 & \multirow[t]{2}{*}{858} \\
\hline GCK Ex_12.1R & CCAGAATCACAAGCCACTCA & 20 & \\
\hline GCK Ex_12.3F & CAGTCCTGGCCATTTTCTTG & 20 & \multirow[t]{2}{*}{772} \\
\hline GCK Ex_12.3R & TGCATGTGACGCAGACTCTT & 20 & \\
\hline
\end{tabular}

Table 2- Microsatellite Markers used to test linkage to known loci of MODY

\begin{tabular}{|c|c|c|c|c|c|}
\hline $\begin{array}{l}\text { MODY } \\
\text { Type }\end{array}$ & Gene & Chromosome & Chromosome Location & $\begin{array}{l}\text { Microsatellite } \\
\text { Marker }\end{array}$ & $\begin{array}{l}\text { Chromosomal } \\
\text { Location }\end{array}$ \\
\hline \multirow[t]{2}{*}{ MODY 1} & HNF4A & 2 & $1.82 E+08$ & D2S1776 & $1.7 \mathrm{E}+08$ \\
\hline & & & & D2S1391 & $1.85 \mathrm{E}+08$ \\
\hline \multirow[t]{2}{*}{ MODY 2} & GCK & 7 & $44150395-44195563$ & D7S2846 & 378755 \\
\hline & & & & D7S1818 & 49133605 \\
\hline \multirow[t]{2}{*}{ MODY 3} & HNF1A & 12 & $1.2 \mathrm{E}+08$ & D12S395 & $1.19 \mathrm{E}+08$ \\
\hline & & & & D12S2078 & $1.26 \mathrm{E}+08$ \\
\hline \multirow[t]{2}{*}{ MODY 4} & IPF1, PDX1 & 13 & $27392157-27397394$ & D13S787 & 22178721 \\
\hline & & & & D13S1493 & 31806690 \\
\hline \multirow[t]{2}{*}{ MODY 5} & HNF1B & 17 & $33120548-33179182$ & D17S1293 & 32705555 \\
\hline & & & & D17S1299 & 39367471 \\
\hline \multirow[t]{2}{*}{ MODY 6} & $\begin{array}{l}\text { Neurod1, } \\
\text { BETA2 }\end{array}$ & 2 & $182249440-182253626$ & D2S1360 & 17476434 \\
\hline & & & & D2S405 & 29451166 \\
\hline
\end{tabular}


Table 3- PCR conditions for Exclusion Study

\begin{tabular}{|c|c|c|}
\hline \multicolumn{3}{|c|}{$\begin{array}{c}\text { MODY 901-922, Marker D2S1776 } \\
\mathrm{R}^{\star} \mathrm{n} / \mathrm{Vol}=15 \text { microlitre }\end{array}$} \\
\hline PCR Reagents & $\mathrm{R}^{*} \mathrm{n} / \mathrm{Vol}$ & $24 \mathrm{R}^{*} \mathrm{n} / \mathrm{Vol}$ \\
\hline Deionized water & 5 & 120 \\
\hline 10X Buffer & 1 & 24 \\
\hline dNTPs & 1 & 24 \\
\hline Taq polymerase & 0.2 & 4.8 \\
\hline Forward Primer & 0.3 & 7.2 \\
\hline Reverse Primer & 0.3 & 7.2 \\
\hline Template DNA & 1.5 & 1.5 \\
\hline \multicolumn{3}{|c|}{$\begin{array}{c}\text { MODY 901-922, Marker D7S2846 } \\
\mathrm{R}^{*} \mathrm{n} / \mathrm{Vol}=15 \text { microlitre } \\
\end{array}$} \\
\hline PCR Reagents & $\mathrm{R}^{*} \mathrm{n} / \mathrm{Vol}$ & $28 R^{*} n / \mathrm{Vol}$ \\
\hline Deionized water & 5 & 140 \\
\hline 10X Buffer & 1 & 28 \\
\hline dNTPs & 1 & 28 \\
\hline Taq polymerase & 0.2 & 5.6 \\
\hline Forward Primer & 0.3 & 8 \\
\hline Reverse Primer & 0.3 & 8 \\
\hline Template DNA & 1.5 & 1.5 \\
\hline \multicolumn{3}{|c|}{$\begin{array}{c}\text { MODY 901-922, Marker D7S1818 } \\
\mathrm{R}^{*} \mathrm{n} / \mathrm{Vol}=15 \text { microlitre } \\
\end{array}$} \\
\hline PCR Reagents & $\mathrm{R}^{*} \mathrm{n} / \mathrm{Vol}$ & $28 R^{*} n / \mathrm{Vol}$ \\
\hline Deionized water & 5 & 140 \\
\hline 10X Buffer & 1 & 28 \\
\hline dNTPs & 1 & 28 \\
\hline Taq polymerase & 0.2 & 5.6 \\
\hline Forward Primer & 0.3 & 8 \\
\hline Reverse Primer & 0.3 & 8 \\
\hline Template DNA & 1.5 & 1.5 \\
\hline \multicolumn{3}{|c|}{$\begin{array}{c}\text { MODY 901-922, Marker D12S395 } \\
\mathrm{R}^{*} \mathrm{n} / \mathrm{Vol}=15 \text { microlitre }\end{array}$} \\
\hline PCR Reagents & $\mathrm{R}^{*} \mathrm{n} / \mathrm{Vol}$ & $25 R^{*} n / \mathrm{Vol}$ \\
\hline Deionized water & 5 & 125 \\
\hline 10X Buffer & 1 & 25 \\
\hline dNTPs & 1 & 25 \\
\hline Taq polymerase & 0.2 & 5 \\
\hline Forward Primer & 0.3 & 7 \\
\hline Reverse Primer & 0.3 & 7 \\
\hline Template DNA & 1.5 & 1.5 \\
\hline \multicolumn{3}{|c|}{$\begin{array}{c}\text { MODY 901-922, Marker D12S2078 } \\
\mathrm{R}^{*} \mathrm{n} / \mathrm{Vol}=15 \text { microlitre }\end{array}$} \\
\hline $\begin{array}{l}\text { PCR Reagents } \\
\end{array}$ & $\mathrm{R}^{*} \mathrm{n} / \mathrm{Vol}$ & $25 R^{*} \mathrm{n} / \mathrm{Vol}$ \\
\hline Deionized water & 5 & 125 \\
\hline 10X Buffer & 1 & 25 \\
\hline dNTPs & 1 & 25 \\
\hline Taq polymerase & 0.2 & 5 \\
\hline Forward Primer & 0.3 & 7 \\
\hline Reverse Primer & 0.3 & 7 \\
\hline Template DNA & 1.5 & 1.5 \\
\hline \multicolumn{3}{|c|}{$\begin{array}{c}\text { MODY 901-922, Marker D13S787 } \\
\mathrm{R}^{*} \mathrm{n} / \mathrm{Vol}=15 \text { microlitre }\end{array}$} \\
\hline $\begin{array}{l}\text { PCR Reagents } \\
\end{array}$ & $\mathrm{R}^{*} \mathrm{n} / \mathrm{Vol}$ & $24 \mathrm{R}^{\star} \mathrm{n} / \mathrm{Vol}$ \\
\hline Deionized water & 5 & 120 \\
\hline 10X Buffer & 1 & 24 \\
\hline
\end{tabular}




\begin{tabular}{|c|c|c|}
\hline dNTPs & 1 & 24 \\
\hline Taq polymerase & 0.2 & 4.8 \\
\hline Forward Primer & 0.3 & 7.2 \\
\hline Reverse Primer & 0.3 & 7.2 \\
\hline Template DNA & 1.5 & 1.5 \\
\hline \multicolumn{3}{|c|}{$\begin{array}{l}\text { MODY 901-922, Marker D13S1493 } \\
R^{*} \mathrm{n} / \mathrm{Vol}=15 \text { microlitre }\end{array}$} \\
\hline PCR Reagents & $\mathrm{R}^{*} \mathrm{n} / \mathrm{Vol}$ & $24 R^{*} n / \mathrm{Vol}$ \\
\hline Deionized water & 2.7 & 64.8 \\
\hline 2*DyNazyme & 5 & 120 \\
\hline Forward Primer & 0.4 & 9.6 \\
\hline Reverse Primer & 0.4 & 9.6 \\
\hline Template DNA & 1.5 & 1.5 \\
\hline \multicolumn{3}{|c|}{$\begin{array}{c}\text { MODY 901-922 } \\
\text { Marker D17S1293 } \\
\mathrm{R}^{\star} \mathrm{n} / \mathrm{Vol}=15 \text { microlitre }\end{array}$} \\
\hline PCR Reagents & $\mathrm{R}^{*} \mathrm{n} / \mathrm{Vol}$ & $28 R^{*} \mathrm{n} / \mathrm{Vol}$ \\
\hline Deionized water & 5 & 140 \\
\hline 10X Buffer & 1 & 28 \\
\hline dNTPs & 1 & 28 \\
\hline Taq polymerase & 0.2 & 5.6 \\
\hline Forward Primer & 0.3 & 8 \\
\hline Reverse Primer & 0.3 & 8 \\
\hline Template DNA & 1.5 & 1.5 \\
\hline \multicolumn{3}{|c|}{$\begin{array}{l}\text { MODY 901-922, Marker D17S1299 } \\
R^{*} n / \text { Vol }=15 \text { microlitre }\end{array}$} \\
\hline PCR Reagents & $\mathrm{R}^{*} \mathrm{n} / \mathrm{Vol}$ & $28 \mathrm{R}^{*} \mathrm{n} / \mathrm{Vol}$ \\
\hline Deionized water & 5 & 140 \\
\hline 10X Buffer & 1 & 28 \\
\hline dNTPs & 1 & 28 \\
\hline Taq polymerase & 0.2 & 5.6 \\
\hline Forward Primer & 0.3 & 8 \\
\hline Reverse Primer & 0.3 & 8 \\
\hline Template DNA & 1.5 & 1.5 \\
\hline \multicolumn{3}{|c|}{$\begin{array}{c}\text { MODY 901-922, Marker D2S1360 } \\
\mathrm{R}^{*} \mathrm{n} / \mathrm{Vol}=15 \text { microlitre }\end{array}$} \\
\hline PCR Reagents & $\mathrm{R}^{*} \mathrm{n} / \mathrm{Vol}$ & $32 R^{*} n / \mathrm{Vol}$ \\
\hline Deionized water & 5 & 160 \\
\hline 10X Buffer & 1 & 32 \\
\hline dNTPs & 1 & 32 \\
\hline Taq polymerase & 0.2 & 6.4 \\
\hline Forward Primer & 0.3 & 8 \\
\hline Reverse Primer & 0.3 & 8 \\
\hline Template DNA & 1.5 & 1.5 \\
\hline \multicolumn{3}{|c|}{$\begin{array}{l}\text { MODY 901-922, Marker D2S405 } \\
R^{*} \mathrm{n} / \mathrm{Vol}=15 \text { microlitre }\end{array}$} \\
\hline $\begin{array}{l}\text { PCR Reagents } \\
\end{array}$ & $\mathrm{R}^{\star} \mathrm{n} / \mathrm{Vol}$ & $24 R^{*} \mathrm{n} / \mathrm{Vol}$ \\
\hline Deionized water & 5 & 120 \\
\hline 10X Buffer & 1 & 24 \\
\hline dNTPs & 1 & 24 \\
\hline Taq polymerase & 0.2 & 4.8 \\
\hline Forward Primer & 0.3 & 7.2 \\
\hline Reverse Primer & 0.3 & 7.2 \\
\hline Template DNA & 1.5 & 1.5 \\
\hline
\end{tabular}




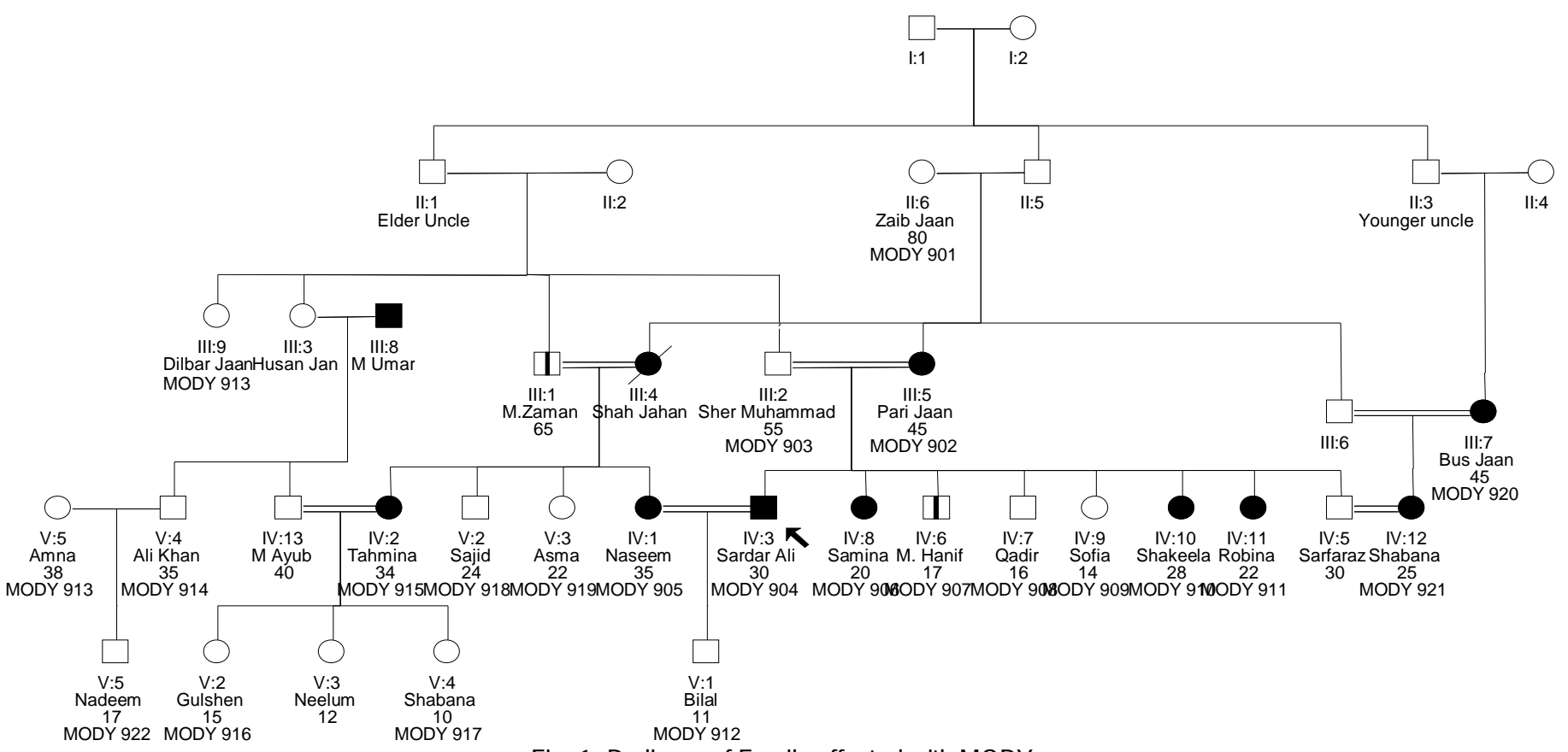

Fig. 1- Pedigree of Family affected with MODY

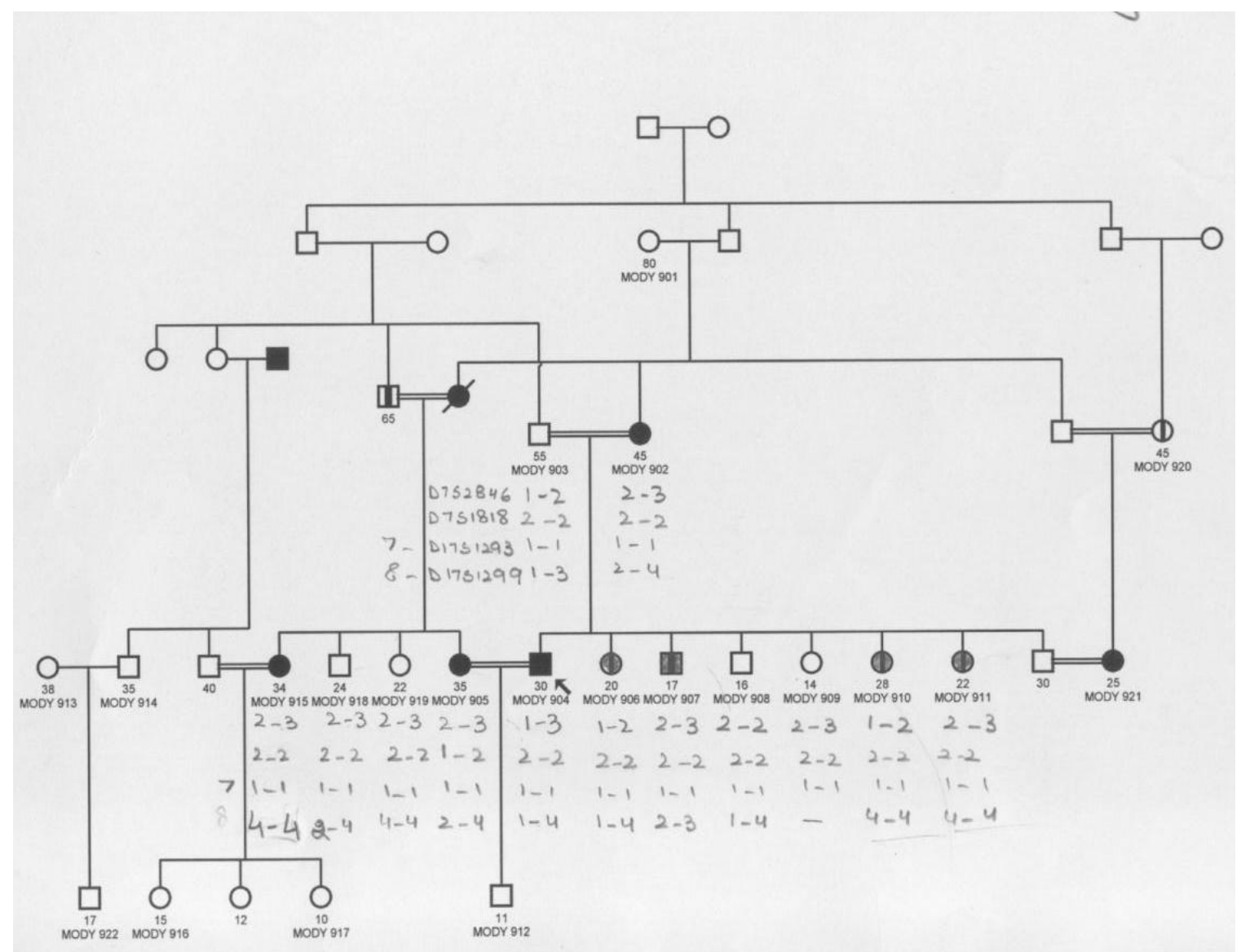

Fig. 2- Exclusion study of the family to six known loci for MODY 


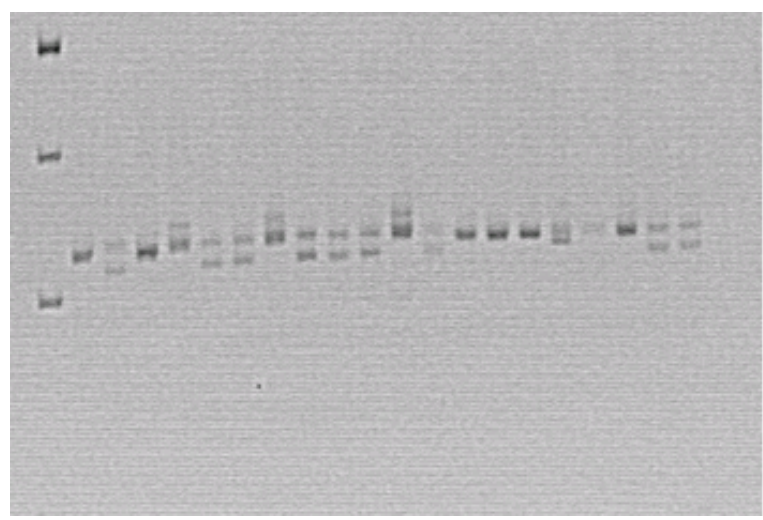

D2S1776

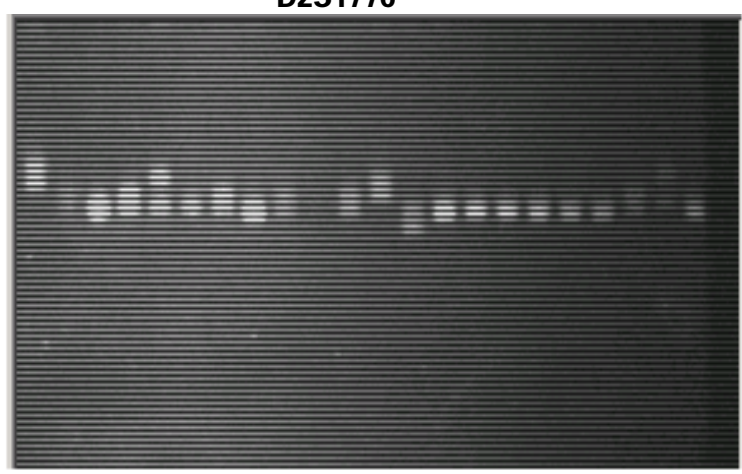

D7S1818

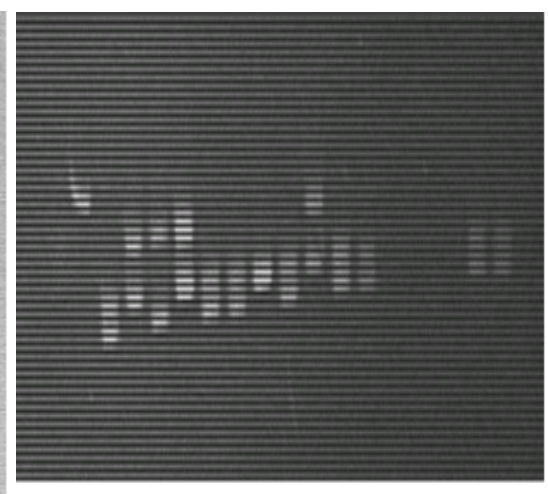

D7S2846

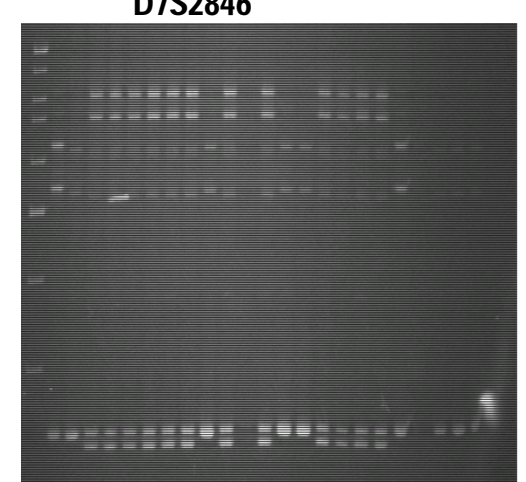

D12S395

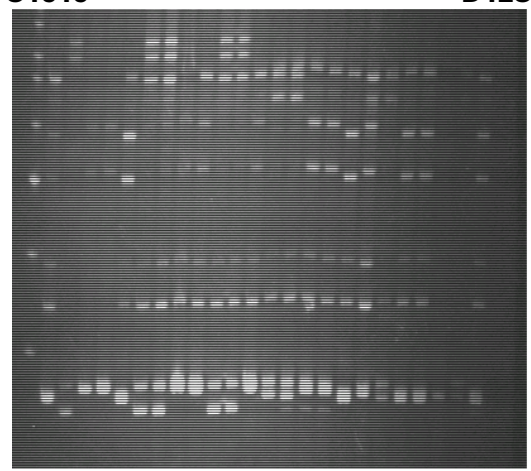

D12S2078

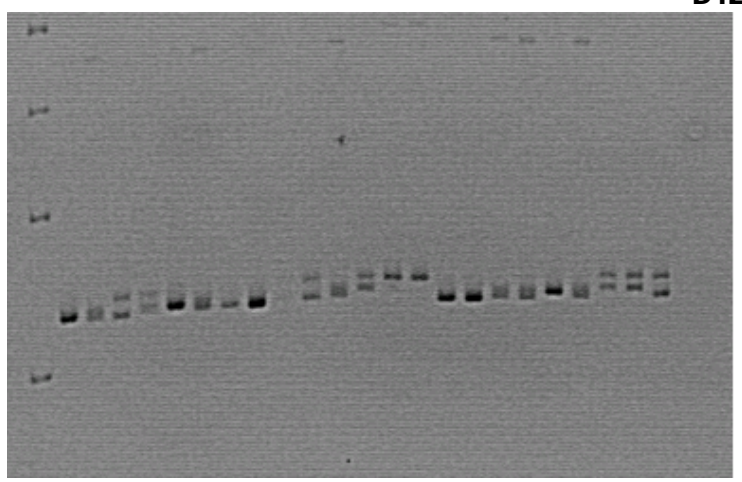

D13S1493

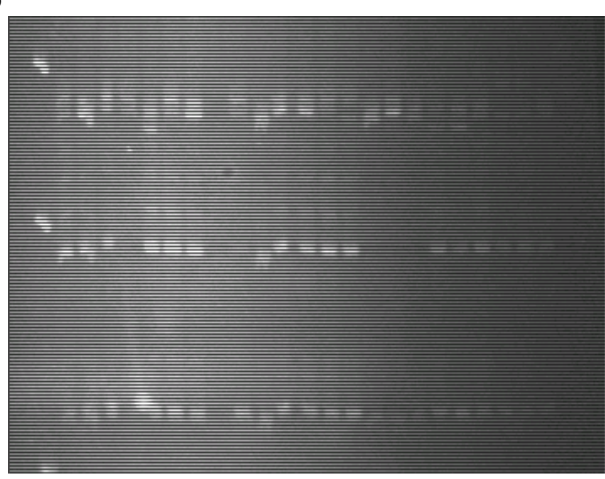

D17S1293 


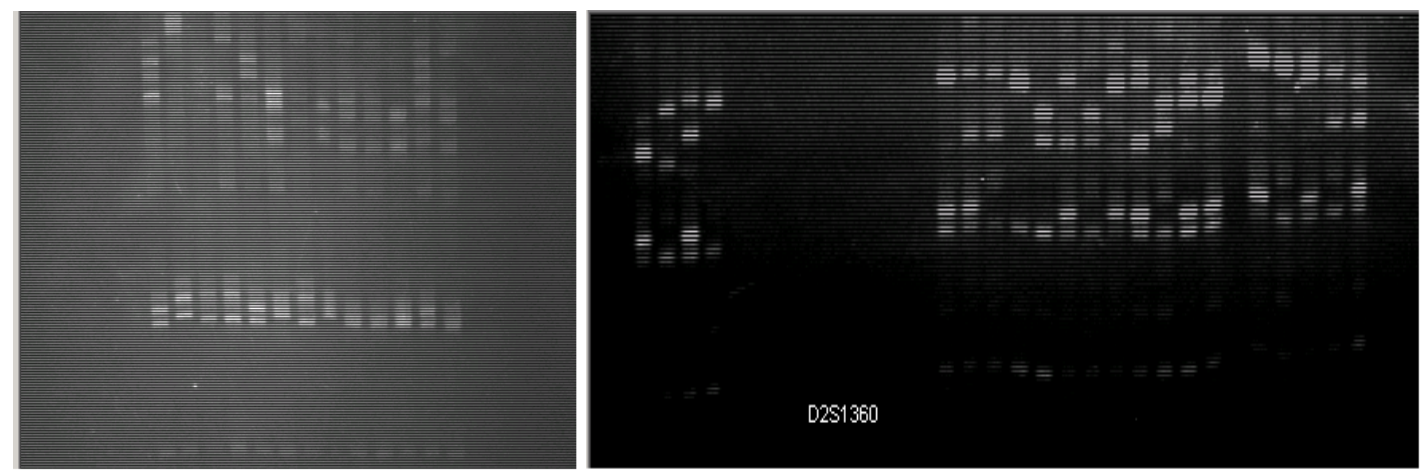

D17S1299

D2S1360

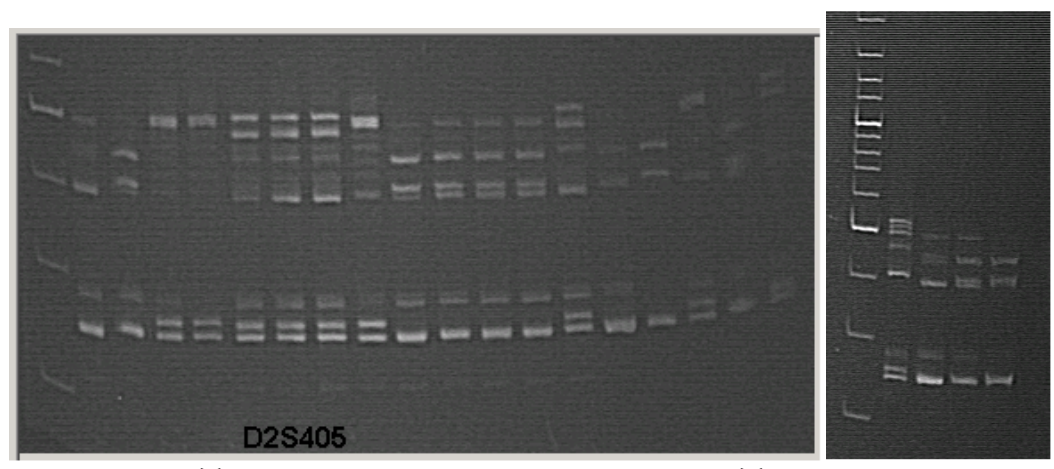

(a), D2S405

(b), D2S405

Fig. 3- Gel results of exclusion study or linkage analysis

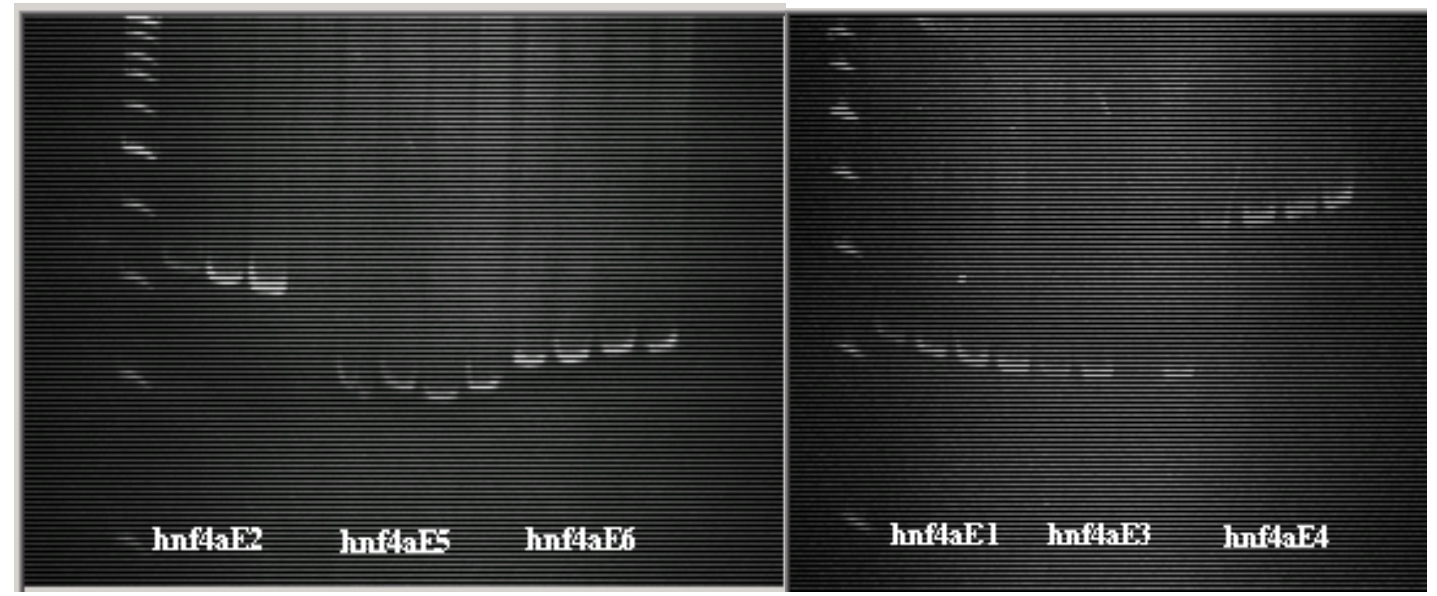

HNF4-Alpha Ex1, Ex2, Ex3, Ex4, Ex5, Ex6 


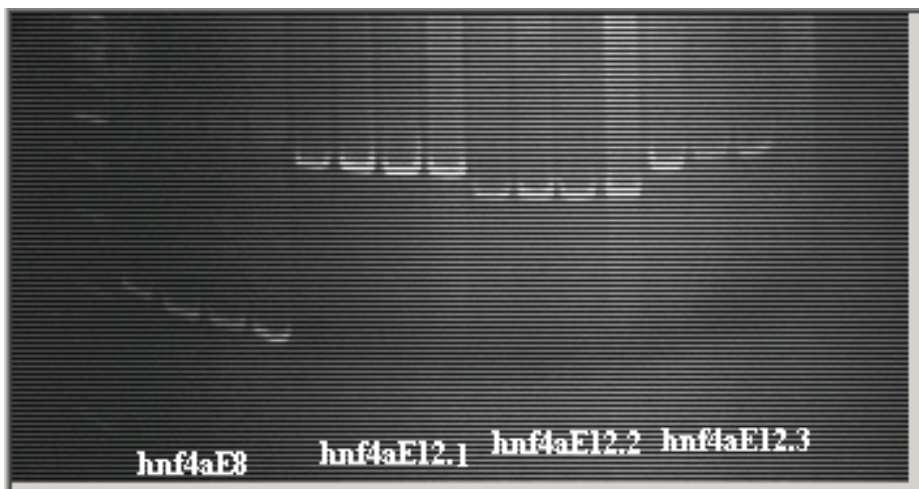

HNF4-Alpha Ex8, Ex12.1, Ex12.2, Ex12.3

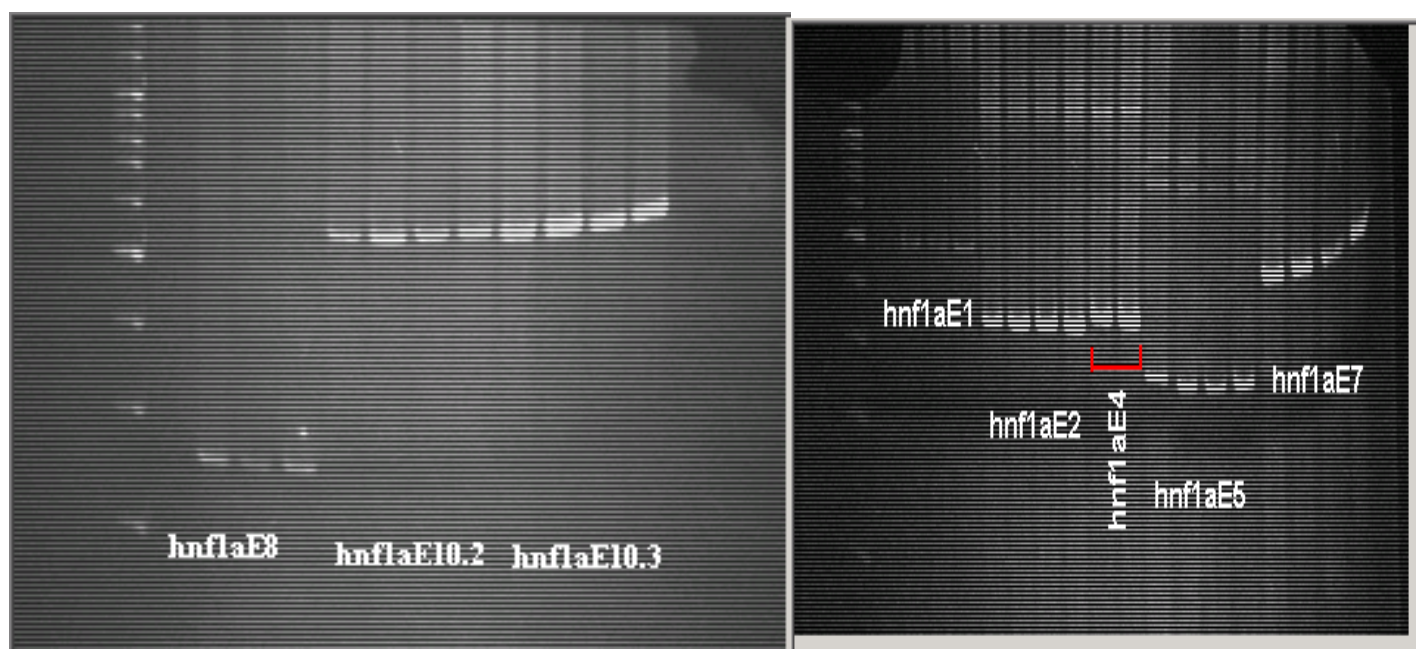

HNF1-Alpha Ex1, Ex2, Ex4, Ex5, Ex7, Ex8, Ex 10.2, Ex10.3

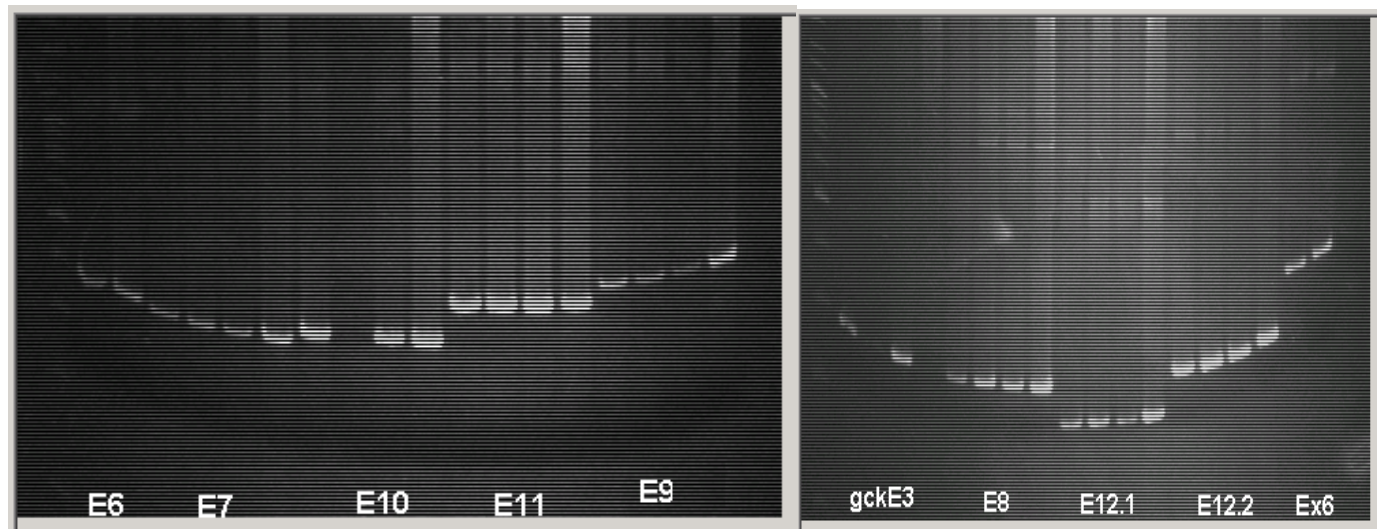

GCK Ex3, Ex6, Ex7, Ex8, Ex9, Ex10, Ex11, Ex12.1, Ex12.2

Fig. 4- Gel results of SSCP 